\title{
Research Article \\ Optimal Skip Entry Trajectory for Lunar Return Vehicle with Trim-Flaps
}

\author{
Yin Wang ${ }^{1}$ and Keming Yao \\ ${ }^{1}$ College of Electric and Information Engineering, Taiyuan University of Science and Technology, Taiyuan 312400, China \\ ${ }^{2}$ College of Electric and Information Engineering, Jiangsu University of Technology, Changzhou 213001, China \\ Correspondence should be addressed to Yin Wang; xpw417@163.com
}

Received 1 December 2016; Accepted 16 January 2017; Published 6 February 2017

Academic Editor: Stefan Balint

Copyright (c) 2017 Yin Wang and Keming Yao. This is an open access article distributed under the Creative Commons Attribution License, which permits unrestricted use, distribution, and reproduction in any medium, provided the original work is properly cited.

A medium lift-to-drag ratio lunar return vehicle with trim-flaps is presented in this paper. The trajectory optimization design under heat-rate constrain for skip entry lunar return vehicle is analyzed. The optimization problem with a first-order state constraint is introduced. The trajectory applying the Pontryagin maximum principle under the performance of minimum heat is optimized, and the optimal expression of lift coefficient is derived. The simulation studies show that this research method can decrease the heat-rate effectively.

\section{Introduction}

When the lunar return vehicle reenters the atmosphere, its speed is up to $11 \mathrm{~km} / \mathrm{s}$, and its kinetic energy is about twice that of the near-earth orbiter. Low lift-drag ratio lunar return vehicle, such as the Apollo spacecraft, using the way of direct reentry has the large reentry overload and the high rate of heat flow in its return process, so it is not conducive to the safety return of return vehicle. And its poor mobility makes it difficult to achieve precise landing.

Appropriately increasing the return capsule lift-drag ratio and using the way of skip reentry can improve the safety performance of the return vehicle. It also can significantly improve the maneuvering ability of the lunar return vehicle and offer more choices for spacecraft landing site [1-3]. The vehicle with trim-flaps is a kind of medium lift-drag ratio vehicle, which has a simpler structure than that of lift type vehicle. Since the 1990s, scholars like Shui et al. [4-7] have done some studies in conceptual design and return optimal trajectory for vehicles with trim-flaps.

Skip entry is the way that return vehicle flies out of the atmosphere boosted by the lift after the vehicle reentry with a smaller reentry angle and then does some ballistic flight outside the atmosphere; then it reenters the atmosphere again $[7,8]$. Istratie et al. have done a series of studies in the initial reentry optimal trajectory design of the near-earth orbit skip reentry [8-11].

In view of such fact that a lot of research has been done for reentry trajectory optimization design of near-earth orbit returning spacecraft, only initial reentry of vehicle skip reentry is discussed in this paper. A return vehicle with trim-flap is used as the lunar return vehicle model; the optimization problem with a first-order state constraint is introduced; the trajectory applying the Pontryagin maximum principle under the performance of minimum heat is optimized and the optimal expression of lift coefficient is derived in this paper.

\section{Reentry Model of Sample Return Vehicle}

The physical model of return vehicle with trim-flaps is shown in Figure 1.

The maximum lift-drag ratio of vehicle is about 0.7 . At the end of the vehicle there is the aerodynamic wing, and it is used to control the attitude of the vehicle in the reentry process. The lift-drag ratio curve of the vehicle is shown in Figure 2.

In the initial stage of vehicle design, the motion of the center of mass is only needed to be considered in order to understand the aerospace vehicle flight path and flight performance. In this paper, the earth is assumed to be 


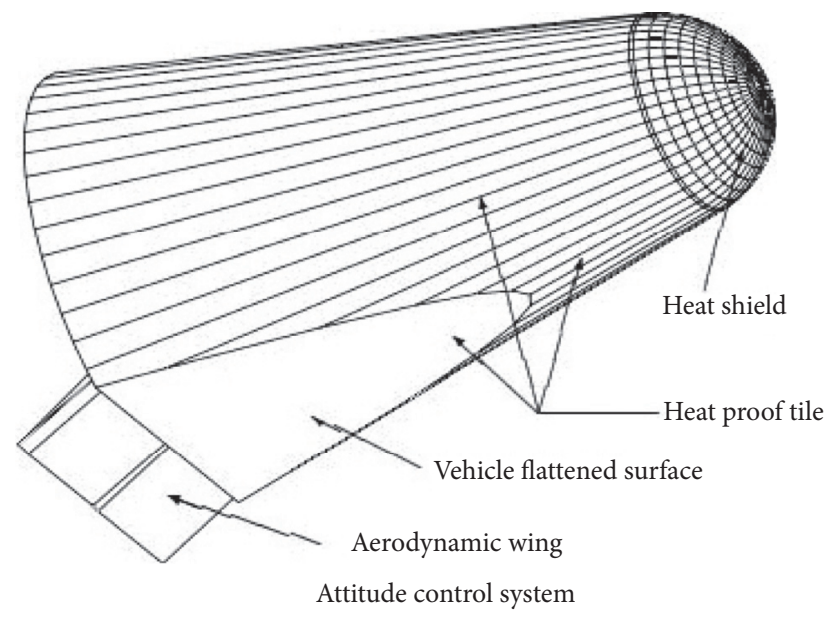

Figure 1: Return vehicle with trim-flaps.
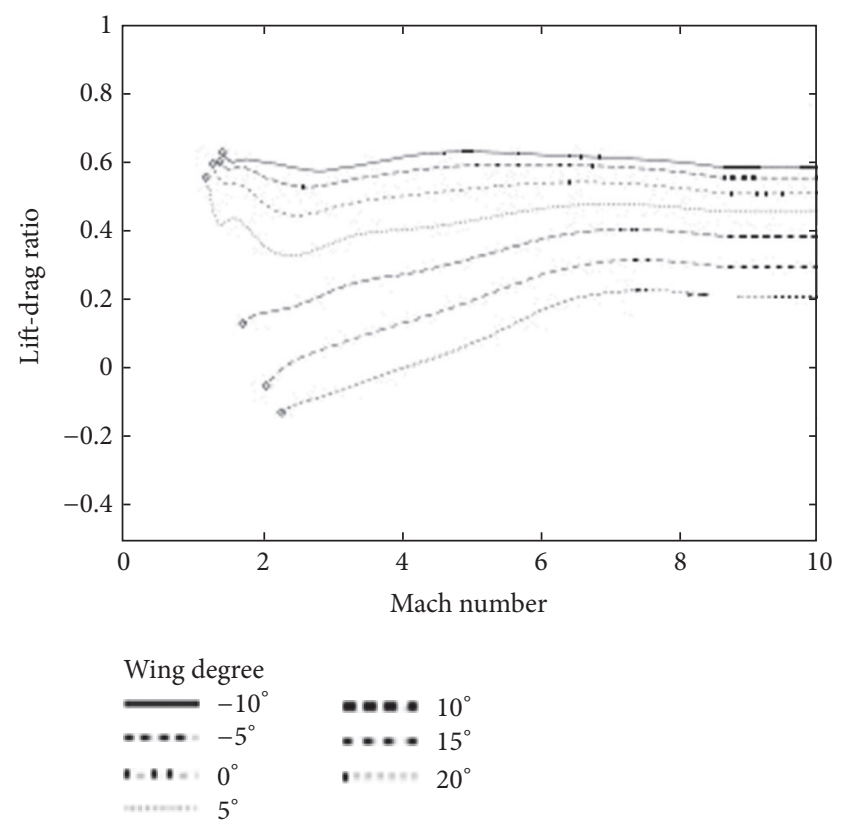

FIgURE 2: Curves of lift-to-drag ratio.

round; only the motion equation of the longitudinal plane is considered. If the control system is in the ideal work, the effect of the attitude control system force can ignored, and the return vehicle is in unpowered reentry, so the equations of motion for vehicle can be described as follows [7-10]:

$$
\begin{aligned}
& \frac{\mathrm{d} v}{\mathrm{~d} t}=-\frac{C_{D} \rho v^{2} A}{2 m}-g \sin \theta, \\
& \frac{\mathrm{d} \theta}{\mathrm{d} t}=\frac{C_{L} \rho v A}{2 m}+\left(\frac{v}{r}-\frac{g}{v}\right) \cos \theta, \\
& \frac{\mathrm{d} h}{\mathrm{~d} t}=v \sin \theta,
\end{aligned}
$$

where $v$ is vehicle speed, $\theta$ is vehicle flight path angle, $r$ is vehicle radial distance, $h$ is vehicle height, $\rho$ is vehicle air density, $g$ is vehicle acceleration of gravity, $m$ is vehicle quality, $A$ is vehicle reference area, $C_{D}$ is vehicle drag coefficient, and $C_{L}$ is vehicle lift coefficient.

And

$$
\begin{aligned}
\rho & =\rho_{0} e^{-\beta h}, \\
C_{D} & =C_{D_{0}}+K C_{L}^{2},
\end{aligned}
$$

where $\rho_{0}$ is sea level atmospheric density and $\beta$ is atmospheric scale height coefficient. For a certain aerospace vehicle, $C_{D}$ and $K$ are constants determined.

The input variable of this control system is vehicle lift coefficient $C_{L}$. When the motion parameters of the vehicle reentry point are known and the control variable $C_{L}$ is determined, the motion equation has a unique solution.

\section{The Description of Optimal Reentry Problem}

In the skip reentry process, there are some effects on the vehicle by the aerodynamic force and the force of gravity, and the attitude is adjusted to change the flight path. After some time of flight, the vehicle escapes the atmosphere by lift, and then it enters the new Kepler orbit. After doing some ballistic movement, it will reenter the atmosphere again.

The attitude of the vehicle in the air flight is adjusted to change its lift-drag ratio. So the optimal flight trajectory can be found, and it can make the vehicle reach the minimum total heat absorption and meets the heat flow constraint.

During the flight, the heat flux equation of vehicle can be given as follows $[9,10]$ :

$$
\dot{Q}=\rho v^{3}
$$

Heat flow constraint is $\dot{Q} \leq \dot{Q}_{\max }$, and it can also be described as follows:

$$
P_{0}=\dot{Q}-\dot{Q}_{\max }
$$

where $P_{0} \leq 0, \dot{Q}_{\max }$ is the maximum heat flow value, and it is also a positive real number.

Because $P_{0}$ does not explicitly include the control parameter $C_{L}$ and $P_{0}$ explicitly includes the control parameter $C_{L}$ on the first derivative of the time, the heat flow constraint is a first-order state variable inequality constraint.

With derivation of this number $P_{0}$, we can obtain

$$
\begin{aligned}
\frac{\mathrm{d} P_{0}}{\mathrm{~d} t} & =\frac{\mathrm{d} \rho v^{3}}{\mathrm{~d} t} \\
& =3 \rho v^{2}\left(-\frac{C_{D} \rho v^{2} A}{2 m}-\left(g+\frac{\beta v^{2}}{3}\right) \sin (\theta)\right) ;
\end{aligned}
$$

$S$ is defined as

$$
S=-\frac{C_{D} \rho v^{2} A}{2 m}-\left(g+\frac{1}{3} \beta v^{2}\right) \sin \theta .
$$

During the flight, the lift coefficient constraint is

$$
-C_{L \max } \leq C_{L} \leq C_{L \max },
$$


where $C_{L \max }$ is the maximum lift coefficient, and it is also a positive real number.

The optimization objective function is the relative value of the total heat absorption. It can be described as follows:

$$
Q=\int_{0}^{t_{f}} \rho v^{3} \mathrm{~d} t
$$

The initial state of the vehicle is

$$
\begin{aligned}
& v\left(t_{0}\right)=v_{0}, \\
& \theta\left(t_{0}\right)=\theta_{0}, \\
& h\left(t_{0}\right)=h_{0} .
\end{aligned}
$$

The terminal constraint of the vehicle is

$$
\begin{aligned}
& v\left(t_{f}\right)=v_{f}, \\
& h\left(t_{f}\right)=h_{f}, \\
& \theta\left(t_{f}\right)=\theta_{f} .
\end{aligned}
$$

The optimal control problem under the heat flow constraints can be described as follows: constraints (1), (4), and (7) should be met during the flight; constraint (9) should be met at the initial moment; constraint (10) should be met at the terminal time. Under the condition that all of the above constraints are met, the minimum problem of the performance index $Q$ is to be researched.

\section{Optimal Trajectory Design}

According to Pontryagin maximum principle, Hamiltonian can be obtained as follows [10-13]:

$$
H=\rho v^{3}+\lambda_{v} \frac{\mathrm{d} v}{\mathrm{~d} t}+\lambda_{\theta} \frac{\mathrm{d} \theta}{\mathrm{d} t}+\lambda_{h} \frac{\mathrm{d} h}{\mathrm{~d} t},
$$

where $\lambda_{v}, \lambda_{\theta}, \lambda_{h}$ are adjoint variables.

The adjoint equations are described as follows:

$$
\begin{aligned}
& \frac{\mathrm{d} \lambda_{v}}{\mathrm{~d} t}=-\frac{\partial H}{\partial v}-\mu \frac{\partial S}{\partial v}, \\
& \frac{\mathrm{d} \lambda_{\theta}}{\mathrm{d} t}=-\frac{\partial H}{\partial \theta}-\mu \frac{\partial S}{\partial \theta}, \\
& \frac{\mathrm{d} \lambda_{h}}{\mathrm{~d} t}=-\frac{\partial H}{\partial h}-\mu \frac{\partial S}{\partial h},
\end{aligned}
$$

where $\mu$ is Kuhn-Tucker multiplier.

According to the literature [12], the optimal curve can be divided into the free section $\left[t_{0}, t_{1}\right)$, the constraint section $\left[t_{1}, t_{2}\right]$, and the free section $\left(t_{2}, t_{f}\right] . t_{1}$ is the time to enter the restriction moment. $t_{2}$ is the time to exit the restriction moment.

In the free section, $P_{0}<0, \mu=0$, according to $\partial H / \partial C_{L}=$ 0 , we have

$$
C_{L}= \begin{cases}\frac{\lambda_{\theta}}{2 K v \lambda_{v}} & 0 \leq\left|C_{L}\right| \leq C_{L \max } \\ C_{L \max } & C_{L}>C_{L \max } \\ -C_{L \max } & C_{L} \prec-C_{L \max } .\end{cases}
$$

In the restricted section, $P_{0}=0, \mu$ must meet $[14,15]$

$$
\begin{aligned}
\frac{\partial H}{\partial C_{L}}+\mu \frac{\partial S}{\partial C_{L}} & =0, \\
\mu & =\frac{\lambda_{\theta}-2 K C_{L} v \lambda_{v}}{2 K C_{L} v} .
\end{aligned}
$$

According to $S=0$, we have

$$
C_{L}=\sqrt{\frac{-2 m\left(g+(1 / 3) \beta v^{2}\right) \sin \theta-\rho v^{2} A C_{D_{0}}}{\rho v^{2} A K}} .
$$

Thus, the adjoint equations can be written as follows:

$$
\begin{aligned}
\frac{\mathrm{d} \lambda_{v}}{\mathrm{~d} t}= & -3 \rho v^{2}+\frac{\rho v A C_{D}}{m} \lambda_{v} \\
& -\left[\frac{\rho A C_{L}}{2 m}+\left(\frac{1}{r}+\frac{g}{v^{2}}\right) \cos \theta\right] \lambda_{\theta}-\sin \theta \lambda_{h} \\
& +\mu\left(\frac{\rho v A C_{D}}{m}+\frac{2}{3} \beta v \sin \theta\right), \\
\frac{\mathrm{d} \lambda_{\theta}}{\mathrm{d} t}= & g \cos \theta \lambda_{v}+\left(\frac{v}{r}-\frac{g}{v}\right) \sin \theta \lambda_{\theta}-v \cos \theta \lambda_{h} \\
& +\mu\left(g+\frac{1}{3} \beta v^{2}\right) \cos \theta, \\
\frac{\mathrm{d} \lambda_{v}}{\mathrm{~d} t} & \beta \rho v^{3}-\left(\frac{\beta \rho v^{2} A C_{D}}{2 m}+\frac{2 g}{r} \sin \theta\right) \lambda_{v} \\
& -\left[\left(\frac{2 g}{r v}-\frac{v}{r^{2}}\right) \cos \theta-\frac{\beta \rho v A C_{L}}{2 m}\right] \lambda_{\theta} \\
& -\mu\left(\frac{\beta \rho v^{2} A C_{D}}{2 m}+\frac{2 g}{r} \sin \theta\right) .
\end{aligned}
$$

At the moment $t_{1}$, we have

$$
\begin{aligned}
\left.P_{0}\right|_{t_{1}} & =0, \\
\left.H\right|_{t_{1}}+\left.H\right|_{t_{1}} & =0, \\
C_{L}\left(t_{1}{ }^{+}\right) & =C_{L}\left(t_{1}{ }^{-}\right), \\
\lambda_{v}\left(t_{1}{ }^{+}\right) & =\lambda_{v}\left(t_{1}{ }^{-}\right)-\left.\eta \frac{\partial P_{0}}{\partial v}\right|_{t_{1}}, \\
\lambda_{\theta}\left(t_{1}{ }^{+}\right) & =\lambda_{\theta}\left(t_{1}{ }^{-}\right)-\left.\eta \frac{\partial P_{0}}{\partial \theta}\right|_{t_{1}}, \\
\lambda_{r}\left(t_{1}{ }^{+}\right) & =\lambda_{r}\left(t_{1}{ }^{-}\right)-\left.\eta \frac{\partial P_{0}}{\partial r}\right|_{t_{1}},
\end{aligned}
$$

where $\eta$ is a real number.

From $\lambda_{v}\left(t_{1}{ }^{+}\right)=\lambda_{v}\left(t_{1}{ }^{-}\right)-\left.\eta\left(\partial P_{0} / \partial v\right)\right|_{t_{1}}$, we have

$$
\eta=\frac{\lambda_{v}\left(t_{1}^{-}\right)-\lambda_{v}\left(t_{1}^{+}\right)}{3 \rho v^{2}} .
$$


According to (13), (15), and (18), we have

$$
\eta=\frac{\lambda_{\theta}\left(t_{1}^{+}\right) / 2 K v C_{L}\left(t_{1}^{+}\right)-\lambda_{v}\left(t_{1}^{+}\right)}{3 \rho v^{2}} .
$$

At the moment $t_{2}$, we have

$$
\begin{aligned}
\left.H\right|_{t_{2}}{ }^{-}-\left.H\right|_{t_{2}} & =0, \\
C_{L}\left(t_{2}{ }^{+}\right) & =C_{L}\left(t_{2}{ }^{-}\right), \\
\lambda_{v}\left(t_{2}{ }^{+}\right) & =\lambda_{v}\left(t_{2}{ }^{-}\right), \\
\lambda_{\theta}\left(t_{2}{ }^{+}\right) & =\lambda_{\theta}\left(t_{2}{ }^{-}\right), \\
\lambda_{r}\left(t_{2}{ }^{+}\right) & =\lambda_{r}\left(t_{2}{ }^{-}\right) .
\end{aligned}
$$

From the above analysis, the optimal reentry problem under the heat flow restriction is the two-point boundary value problem which must meet the connection requirements of the constraints section ends, when the initial state and the terminal state are known. Adjacent extreme method can be used to solve this problem. $t_{2}$ is selected as the initial moment for calculation.

At the moment $t_{2}, v\left(t_{2}\right), \theta\left(t_{2}\right), h\left(t_{2}\right), \lambda_{v}\left(t_{2}\right), \lambda_{\theta}\left(t_{2}\right)$, and $\lambda_{h}\left(t_{2}\right)$ are unknown. According to $P_{0}=0$, vehicle height at this time can be described as follows:

$$
h\left(t_{2}\right)=\frac{\ln \left(\dot{Q}_{\max } / \rho_{0} v^{3}\right)}{-\beta} .
$$

According to $C_{L}\left(t_{2}{ }^{+}\right)=C_{L}\left(t_{2}{ }^{-}\right)$, we have

$$
\begin{aligned}
& \lambda_{\theta}\left(t_{2}\right) \\
& \quad=2 K v \lambda_{v}\left(t_{2}\right) \sqrt{\frac{-2 m\left(g+(1 / 3) \beta v^{2}\right) \sin \theta}{K \rho v^{2} A}-\frac{C_{D_{0}}}{K}} .
\end{aligned}
$$

The value of $\lambda_{h}\left(t_{2}\right)$ can be calculated by adjacent extreme method. $\lambda_{v}\left(t_{2}\right)$ can be retroactively integrated from time $t_{2}$ to time $t_{1}$ to calculate the value $\lambda_{v}\left(t_{1}{ }^{+}\right)$. According to (8), the value of $\eta$ can be calculated. Substituting $\eta$ into (17), the values of $\lambda_{v}\left(t_{1}{ }^{-}\right), \lambda_{\theta}\left(t_{1}{ }^{-}\right)$, and $\lambda_{h}\left(t_{1}{ }^{-}\right)$can also be calculated. Then, reverse operations of integral are done from $t_{1}$, and the end time is determined by the initial state.

\section{Simulation and Analysis}

Calculations are done for two cases in this paper. One is in the case of restricted lunar return vehicle heat flow; the other is just the opposite. Vehicle parameters are given as follows:

$$
\begin{aligned}
& m=600 \mathrm{~kg}, \\
& A=0.754 \mathrm{~m}^{2}, \\
& C_{D_{0}}=0.4, \\
& K=1.11, \\
& C_{L \max }=1.5 .
\end{aligned}
$$

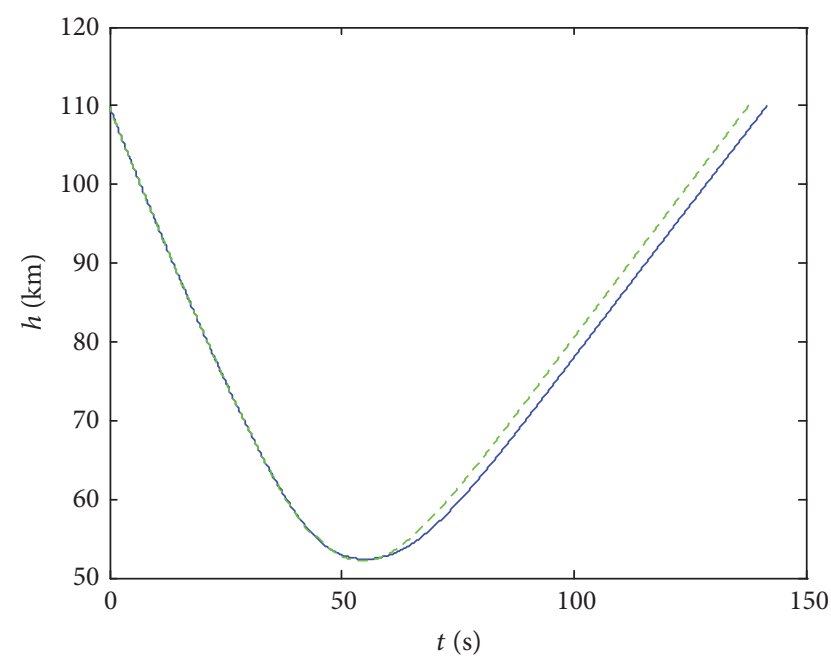

FIgURE 3: Time histories of the altitude.

The initial values are given as follows:

$$
\begin{aligned}
& v(0)=11 \mathrm{~km} / \mathrm{s}, \\
& \theta(0)=-8^{\circ}, \\
& h(0)=110 \mathrm{~km} .
\end{aligned}
$$

A large number of numerical simulations show that the aerospace vehicle has minimum value in the initial reentry. Heat flow constraint value can not take arbitrarily small value. In this paper, heat flow constraint value is given as follows:

$$
\dot{\mathrm{Q}}_{\max }=6.5 \times 10^{4} \mathrm{~W} / \mathrm{cm}^{2} \text {. }
$$

For no power return vehicle, its terminal velocity should be less than $7800 \mathrm{~m} / \mathrm{s}$. In order to make the safe reentry, the secondary reentry angle can not be too large. Thus, the end state is set by

$$
\begin{aligned}
& h\left(t_{f}\right)=110 \mathrm{~km}, \\
& v\left(t_{f}\right)=7750 \mathrm{~m} / \mathrm{s}, \\
& \theta\left(t_{f}\right)=5.7^{\circ} .
\end{aligned}
$$

Through the simulation of optimization model, the actual terminal state is set by

$$
\begin{aligned}
& h\left(t_{f}\right)=110000.2 \mathrm{~m}, \\
& v\left(t_{f}\right)=7753.4 \mathrm{~m} / \mathrm{s}, \\
& \theta\left(t_{f}\right)=5.749^{\circ}, \\
& t_{f}=141.2 \mathrm{~s}, \\
& \Delta t_{2}=3.88 \mathrm{~s} .
\end{aligned}
$$

Curves of vehicle height, velocity, flight path angle, lift coefficient, lift-to-drag ratio, heat flux, pressure, and overload in the atmospheric flight changing with time in the two cases are given by Figures $3-10$. The solid line is the heat constraint optimization curve, and the dashed line is no heat constraint optimization curve in the figures.

During the initial stage with heat constraint, lift coefficient can maintain maximum. This can decrease the speed 


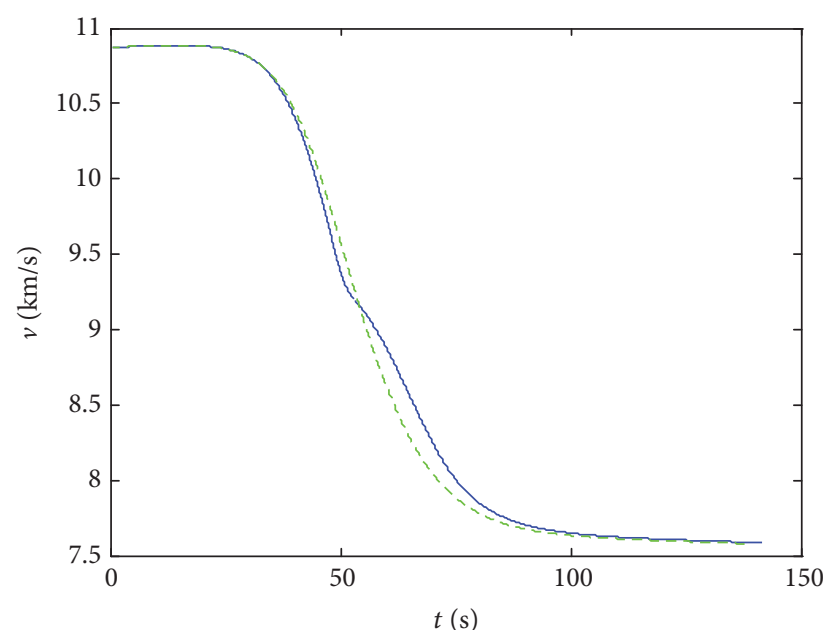

FIgURE 4: Time histories of the velocity.

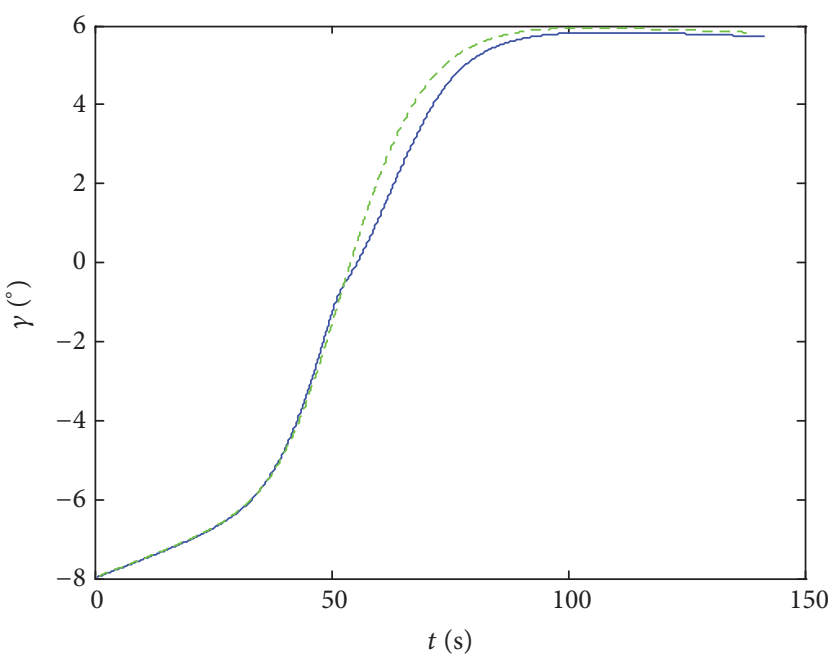

Figure 5: Time histories of the flight path angle.

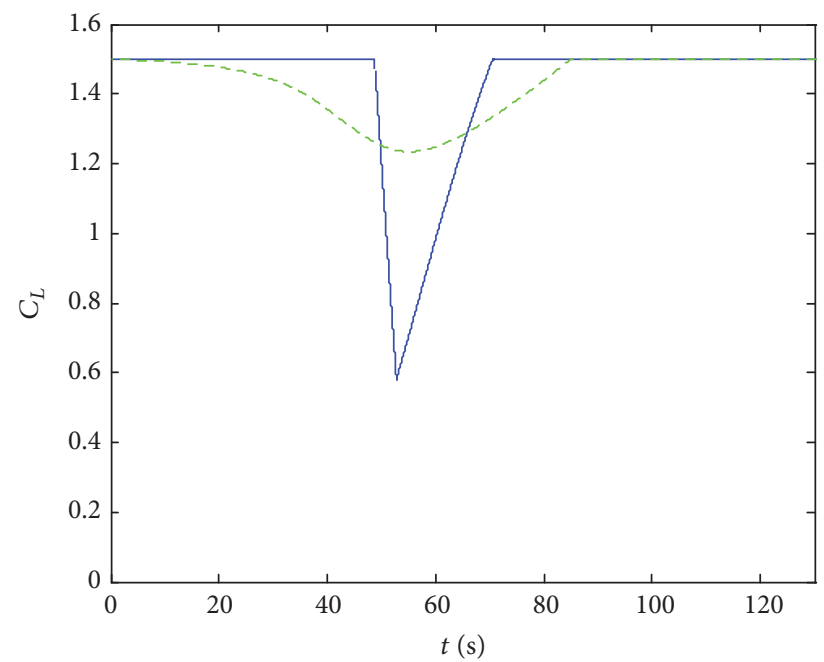

FIgure 6: Time histories of the lift coefficient.

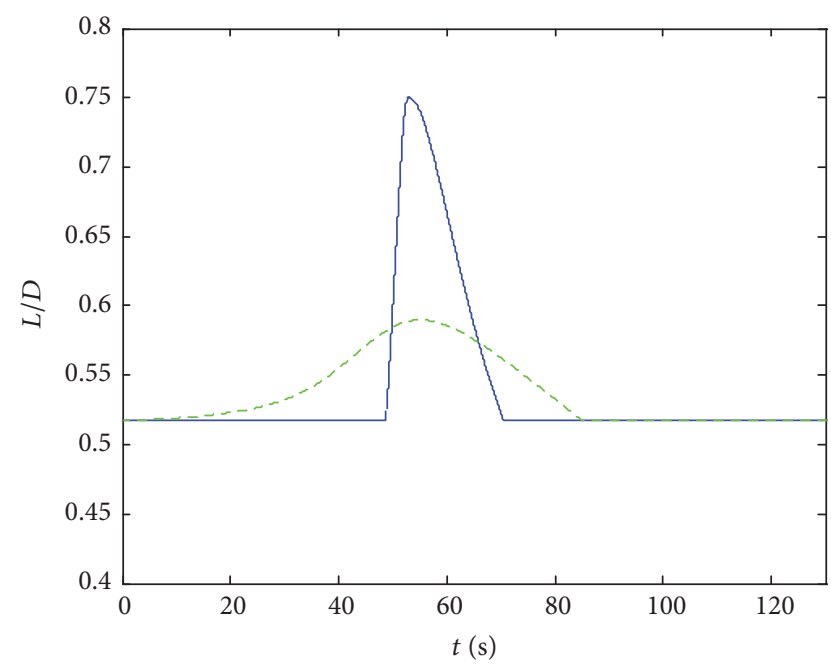

FIGURE 7: Time histories of the lift-drag.

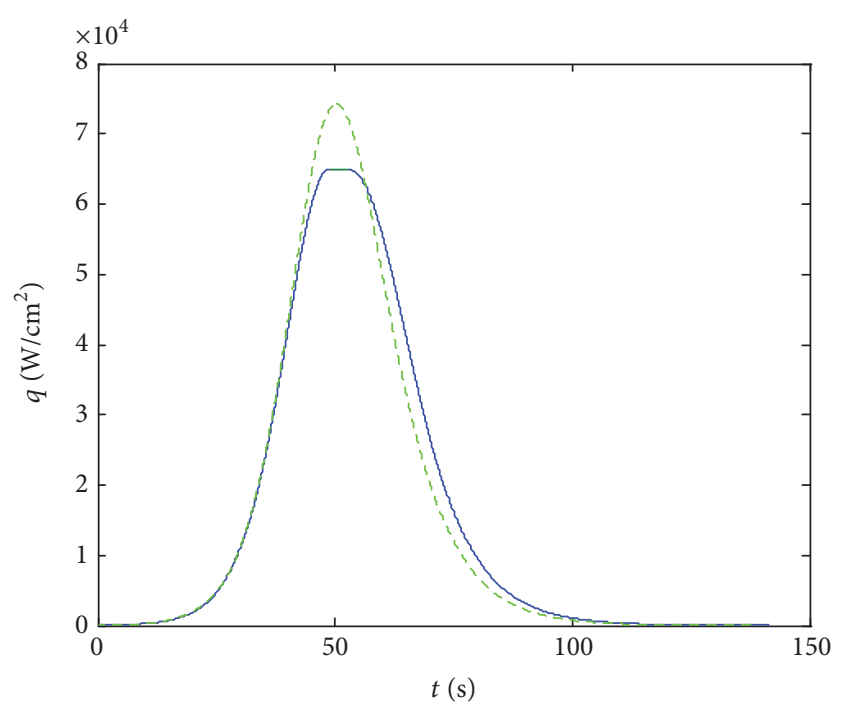

FIGURE 8: Time histories of the heat-rate.

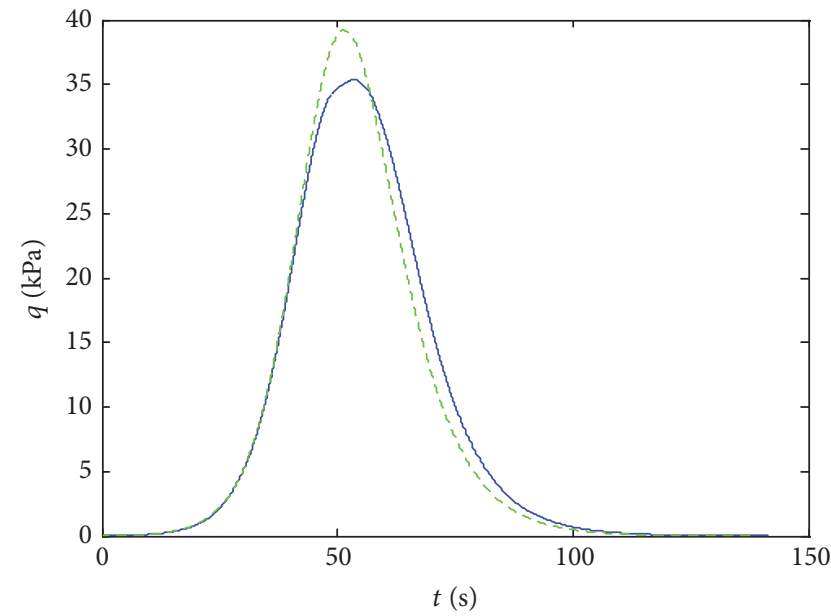

Figure 9: Time histories of the dynamic pressure. 


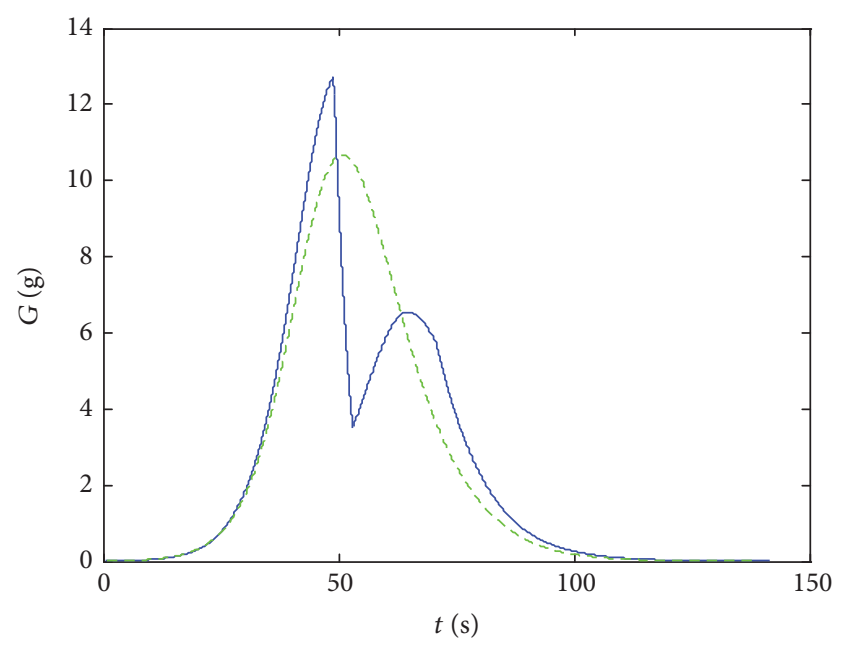

Figure 10: Time histories of the overload.

of the vehicle faster than that in unconstrained case. So it can meet the heat flux constraints during the flight. The maximum dynamic pressure value is smaller than that in unconstrained case, and the maximum dynamic pressure value is greater than that in unconstrained case. The lift coefficient in keeping the maximum value will become smaller after a period of time. This will make the lift-drag ratio become larger. As the reentry vehicle is at low altitude, where the air density is larger, increasing the lift-drag ratio can slow down the speed of the vehicle descent rate. So it can meet the terminal velocity requirement. The flight time in the atmosphere of the return vehicle in heat flow constraint conditions is slightly longer than that in unconstrained conditions.

\section{Conclusion}

A medium lift-to-drag ratio lunar return vehicle with trimflaps is presented as the programming of the lunar return vehicle in this paper. By the numerical simulation above, the following conclusions can be obtained:

(1) In heat flow constraint conditions, the maximum dynamic pressure value of return vehicle is greater than that in unconstrained case. For lunar return vehicle with trim-flaps, the heat flux constraints can not only reduce the quality of thermal protection system of the return vehicle, but also reduce the loads on the control wing.

(2) In heat flow constraint conditions, the maximum overload of the return vehicle is $12.6 \mathrm{~g}$, which is $10.6 \mathrm{~g}$ larger than that in unconstrained case. That is acceptable for the unmanned vehicle. But the maximum overload will need to be constrained for the manned vehicle.

\section{Competing Interests}

The authors declare that they have no competing interests.

\section{Acknowledgments}

This study is supported by the National Natural Science Found of China (Grant no. 91016017), Natural Science Foundation of Shanxi (Grant no. 2015021089), and Doctoral Research Found of Taiyuan University of Science and Technology (Grant no. 20132020).

\section{References}

[1] C. Bai, J. Guo, X. Xu, and G. He, "Robust Skip earth entry guidance for a low L/D spacecraft," in Proceedings of the 12th IEEE International Conference on Mechatronics and Automation (ICMA '15), pp. 1653-1658, Beijing, China, August 2015.

[2] P. Lu, C. Brunner, S. Stachowiak, G. F. Mendeck, M. Tigges, and C. Cerimele, "Verification of a fully numerical entry guidance algorithm," in Proceedings of the AIAA Guidance, Navigation, and Control Conference, pp. 1969-2017, 2016.

[3] F. B. Wang and C. H. Dong, "Reentry trajectory optimization design for lunar return through coevolutionary algorithm," Journal of Beijing University of Aeronautics and Astronautics, vol. 40, no. 5, pp. 629-634, 2014.

[4] Z. Shui, J. Zhou, and Z. Ge, "Lateral entry guidance for lunar return vehicles," Modern Applied Science, vol. 6, no. 3, pp. 100106, 2012.

[5] K. Mohan and M. Patterson, "Optimal trajectory and control generation for landing of multiple aircraft in the presence of obstacles," in Proceedings of the AIAA Guidance, Navigation, and Control Conference, AIAA 2012-4826, Minneapolis, Minn, USA, August 2012.

[6] J. R. French, S. Alan Stern, M. Vozoff, T. McCallum, and C. Deiterich, "Architecture for lunar return using existing assets," Journal of Spacecraft and Rockets, vol. 50, no. 4, pp. 838-847, 2013.

[7] J. Burkhardt, F. Zimmermann, and U. M. Schöttle, “Operational use of guided reentry capsules-system design solutions and mission safety considerations," Aerospace Science and Technology, vol. 8, no. 7, pp. 635-644, 2004.

[8] V. Istratie, “Three-dimensional optimal skip entry with terminal maximum velocity," in Proceedings of the 22nd Atmospheric Flight Mechanics Conference, pp. 19-26, AIAA, New Orleans, La, USA, August 1997.

[9] V. Istratie, "Optimal skip entry with terminal maximum velocity and heat constraint," in Proceedings of the 7th AIAA/ASME Joint Thermophysics and Heat Transfer Conference, Albuquerque, NM, USA, June 1998.

[10] D. Ross and J. Russell, "Mars Ascent Vehicle (MAV): designing for high heritage and low risk," in Proceedings of the 2012 IEEE Aerospace Conference, pp. 1-6, Big Sky, Mont, USA, March 2012.

[11] V. Istratie, "Optimal skip entry into atmosphere with minimum heat and constrains," in AIAA Atmosphere Flight Mechanics Conference, Denver, CO, Aug. 14-17, 2000, Collection of Technical Papers (A00-39676 10-08), pp. 288-294, American Institute of Aeronautics and Astronautis, Reston, Va, USA, 2000.

[12] V. Istratie, "Optimal skip entry into atmosphere with minimum heat," in Proceedings of the 2003 AIAA Atmospheric Flight Mechanics Conference and Exhibit, pp. 11-14, Austin, Tex, USA, August 2003.

[13] H. Seywald, "Variational solutions for the heat-rate-limited aeroassisted orbital transfer problem," Journal of Guidance, Control, and Dynamics, vol. 19, no. 3, pp. 686-692, 1996. 
[14] H. Zhang, J. Zhou, and D. Wu, "The optimal aeroassisted orbital transfer heat flow constraints," Shanghai Spacecraft, no. 4, pp. 611, 1999.

[15] A. Cassioli, D. Izzo, D. Di Lorenzo, and M. Locatelli, "Global optimization approaches for optimal trajectory planning," Modeling and Optimization in Space Engineering, vol. 73, pp. 111-140, 2013. 


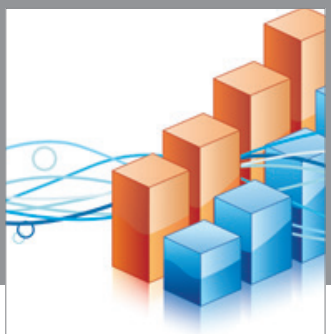

Advances in

Operations Research

vatem alat4

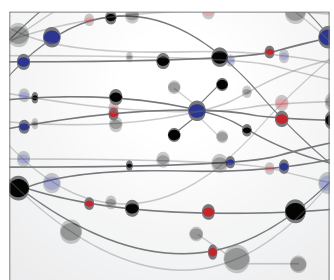

\section{The Scientific} World Journal
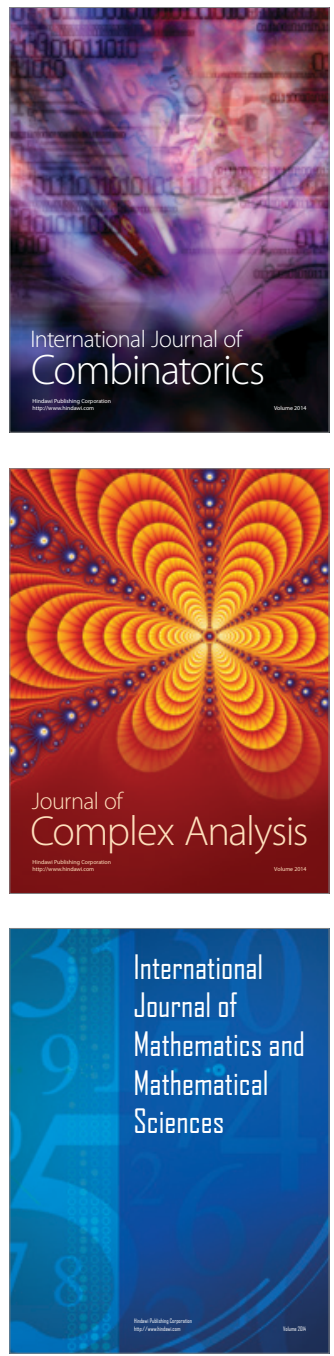
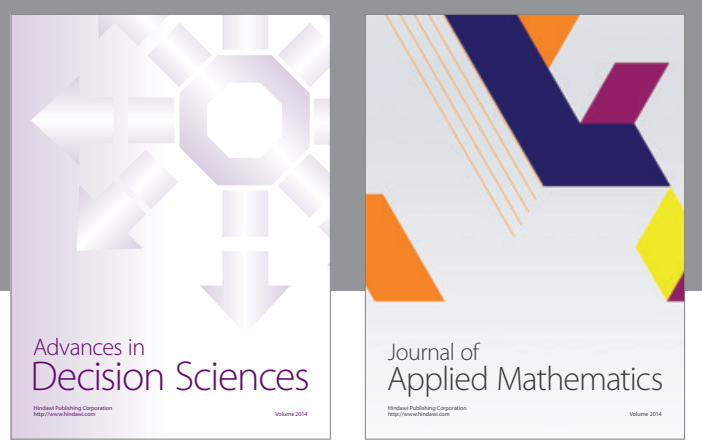

Algebra

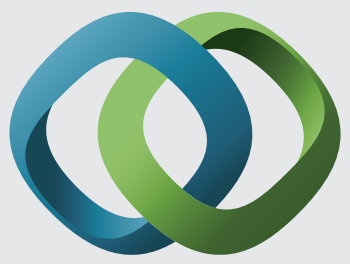

\section{Hindawi}

Submit your manuscripts at

https://www.hindawi.com
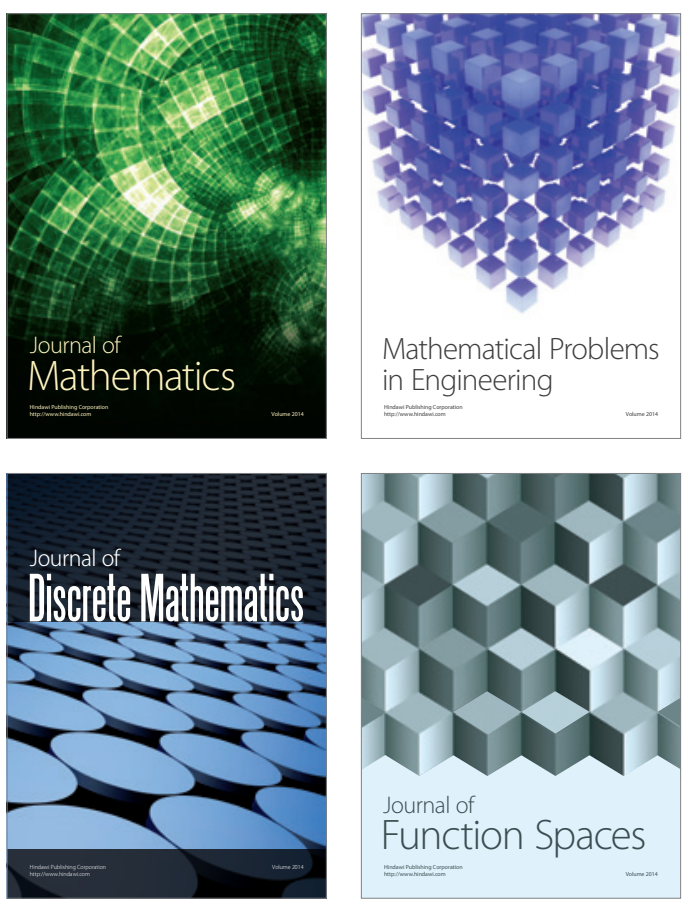

Mathematical Problems in Engineering
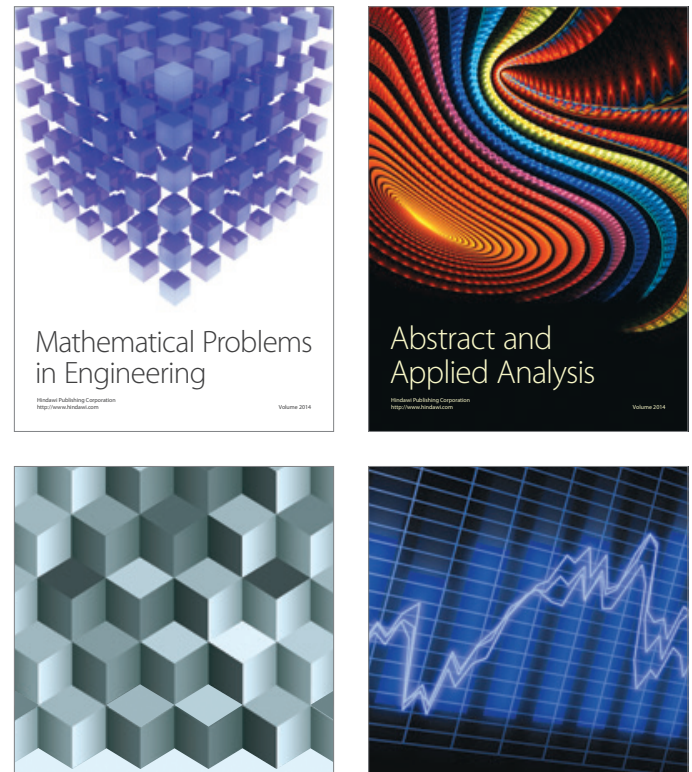

Journal of

Function Spaces

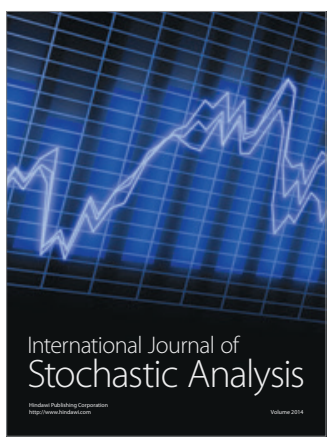

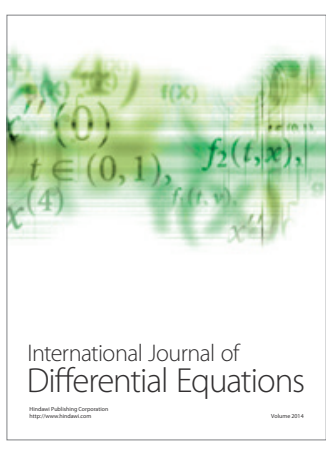
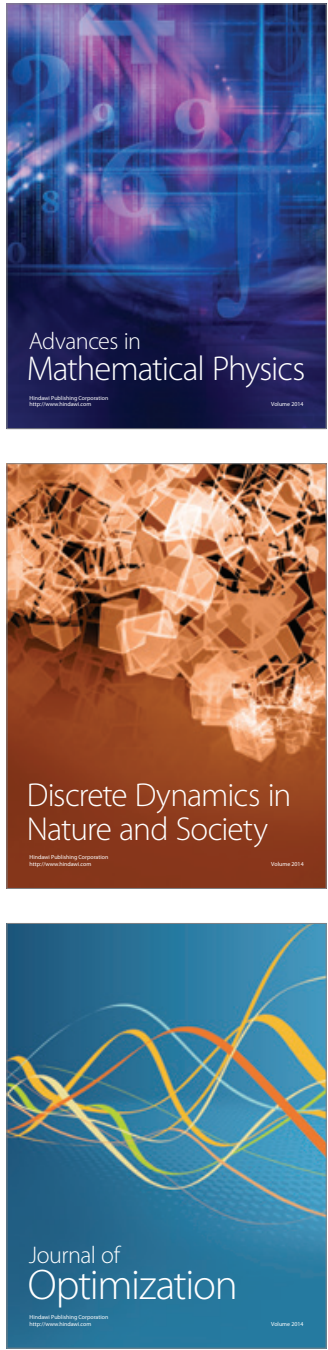